\title{
Erratum to: Design, expression, and characterization of a novel targeted plectasin against methicillin-resistant Staphylococcus aureus
}

\author{
Ruoyu Mao • Da Teng $\cdot$ Xiumin Wang $\cdot$ Di Xi • \\ Yong Zhang • Xiaoyuan Hu • Yalin Yang • Jianhua Wang
}

Published online: 12 March 2013

(C) Springer-Verlag Berlin Heidelberg 2013

\section{Erratum to: Appl Microbiol Biotechnol}

\section{DOI 10.1007/s00253-012-4508-z}

This article unfortunately contained three errors: 1 , In page 3 , the exact size of the pPICAgplectasin plasmid should be "3682 bp" instead of "3138 bp" in Fig 1b; 2, In page 2, the word "General" in note at bottom of Table 1 should be corrected as "Medical"; 3, In page 11, to add "This study is also funded by the Project of National Support Program for Science and Technology in China (no. 2013BAD10B02 and no. 2011BAD26B02)." as the last sentence of "Acknowledgments".

The online version of the original article can be found at http:// dx.doi.org/10.1007/s00253-012-4508-z.

R. Mao $\cdot$ D. Teng $\cdot$ X. Wang $\cdot$ D. Xi $\cdot$ Y. Zhang $\cdot$ X. Hu $\cdot$ Y. Yang $\cdot$ J. Wang

Key Laboratory of Feed Biotechnology, Ministry of Agriculture, Beijing 100081, China

R. Mao $\cdot$ D. Teng $\cdot$ X. Wang $\cdot$ D. Xi $\cdot$ Y. Zhang $\cdot$ X. Hu $\cdot$

J. Wang $(\bowtie)$

Gene Engineering Laboratory, Feed Research Institute, Chinese Academy of Agricultural Sciences, 12 Zhongguancun Nandajie

St., Haidian District,

Beijing 100081, People's Republic of China

e-mail: jhwangfribio@yahoo.com.cn

J. Wang

e-mail:wangjianhua@mail.caas.net.cn 
$\mathbf{a}$

Xho I Kex2

gat tct CTCGAGaaaaga TAC TCT ACT TGT TAC TTT ATT ATG GGT TTT GGT

$\begin{array}{llllllllllll} & Y & S & T & C & Y & F & I & M & G & F & G\end{array}$

TGT AAC GGT CCA TGG GAT GAA GAT GAT ATG CAA TGT CAT AAC CAT

$\begin{array}{llllllllllllllll}C & N & G & P & W & D & E & D & D & M & Q & C & H & N & H\end{array}$

TGT AAG TCT ATT AAG GGT TAC AAG GGT GGT TAC TGT GCT AAG GGT

$\begin{array}{lllllllllllllll}C & K & S & I & K & G & Y & K & G & G & Y & C & A & K & G\end{array}$

GGT TTT GTT TGT AAG TGT TAC TAA TAG GCGGCCGC cagct $t$ t

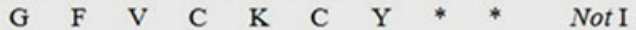

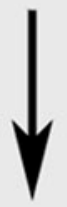

b

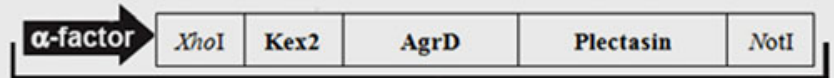

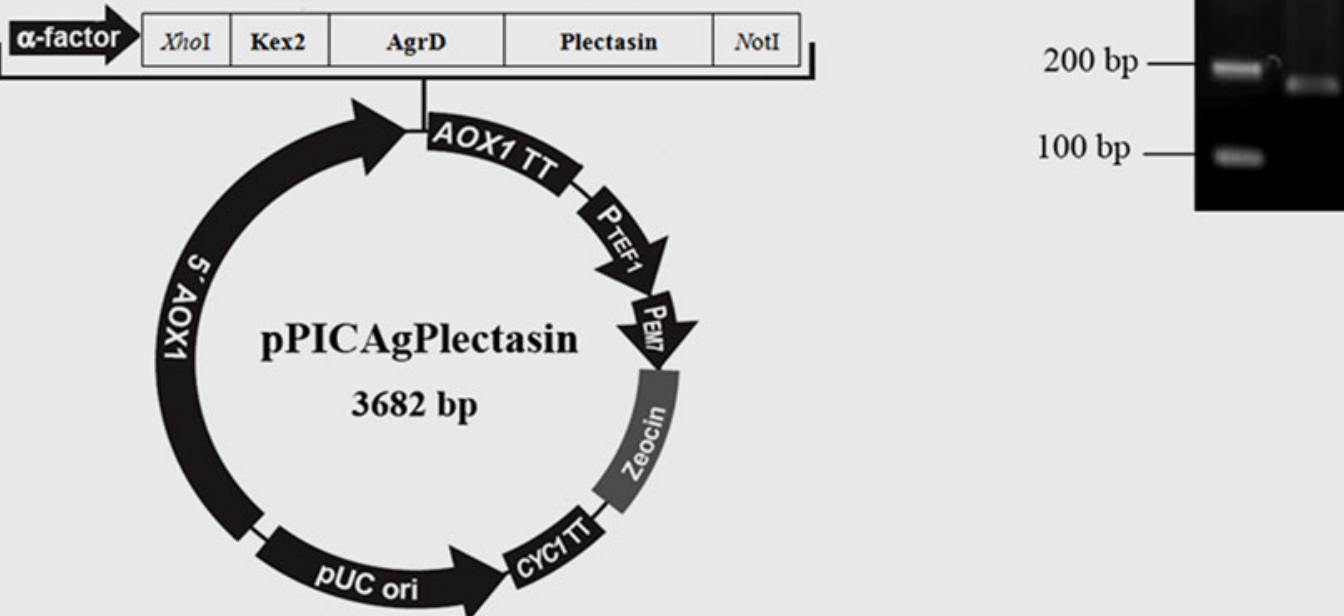

c

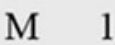

Fig. 1 Construction of the plasmid pPICAgplectasin. a The rAgP nucleotide sequence and its corresponding amino acid sequence. The gray regions indicate the $X h o I$ and NotI restriction endonuclease sites. The underline indicates the Kex 2 cleavage site. The red letters indicate the AgrD1 pheromone nucleotide sequence. $\mathrm{b}$ Schematic diagram of the P. pastoris expression plasmid-pPICAgplectasin. c Electrophoretic analysis of the recombinant vector containing the $\mathrm{rAgP}$ gene. $M$ DNA marker, lane 1 pPICAgplectasin digested by XhoI and NotI 